\title{
Novel ANKRD11 gene mutation in an individual with a mild phenotype of KBG syndrome associated to a GEFS+ phenotypic spectrum: a case report
}

Rita Maria Alves ${ }^{1,2}$, Paolo Uva ${ }^{3}$, Marielza F. Veiga ${ }^{1,4}$, Manuela Oppo ${ }^{5}$, Fabiana C. R. Zschaber ${ }^{2}$, Giampiero Porcu², Henrique P. Porto ${ }^{2}$, Ivana Persico ${ }^{6}$, Stefano Onano ${ }^{5,6}$, Gianmauro Cuccuru ${ }^{3}$, Rossano Atzeni ${ }^{3}$, Lauro C. N. Vieira ${ }^{7}$, Marcos V. A. Pires ${ }^{2,8}$, Francesco Cucca ${ }^{5,6}$, Maria Betânia P. Toralles ${ }^{1}$, Andrea Angius $5^{5,6^{*}}$ (D) and Laura Crisponi $i^{5,6}$

\begin{abstract}
Background: KBG syndrome is a very rare autosomal dominant disorder, characterized by macrodontia, distinctive craniofacial findings, skeletal findings, post-natal short stature, and developmental delays, sometimes associated with seizures and EEG abnormalities. So far, there have been over 100 cases of KBG syndrome reported.

Case presentation: Here, we describe two sisters of a non-consanguineous family, both presenting generalized epilepsy with febrile seizures (GEFS+), and one with a more complex phenotype associated with mild intellectual disability, skeletal and dental anomalies. Whole exome sequencing (WES) analysis in all the family members revealed a heterozygous SCN9A mutation, p.(Lys655Arg), shared among the father and the two probands, and a novel de novo loss of function mutation in the ANKRD11 gene, p.(Tyr1715*), in the proband with the more complex phenotype. The reassessment of the phenotypic features confirmed that the patient fulfilled the proposed diagnostic criteria for KBG syndrome, although complicated by early-onset isolated febrile seizures. EEG abnormalities with or without seizures have been reported previously in some KBG cases.

The shared variant, occurring in SCN9A, has been previously found in several individuals with GEFS+ and Dravet syndrome.

Conclusions: This report describe a novel de novo variant in ANKRD11 causing a mild phenotype of KGB syndrome and further supports the association of monogenic pattern of SCN9A mutations with GEFS+. Our data expand the allelic spectrum of ANKRD11 mutations, providing the first Brazilian case of KBG syndrome. Furthermore, this study offers an example of how WES has been instrumental allowing us to better dissect the clinical phenotype under study, which is a multilocus variation aggregating in one proband, rather than a phenotypic expansion associated with a single genomic locus, underscoring the role of multiple rare variants at different loci in the etiology of clinical phenotypes making problematic the diagnostic path. The successful identification of the causal variant in a gene may not be sufficient, making it necessary to identify other variants that fully explain the clinical picture. The prevalence of blended phenotypes from multiple monogenic disorders is currently unknown and will require a systematic re-analysis of large WES datasets for proper diagnosis in daily practice.
\end{abstract}

Keywords: Whole exome sequencing, KBG syndrome, ANKRD11 gene, Generalized epilepsy with febrile seizures (GEFS+), SCN9A gene

\footnotetext{
* Correspondence: andrea.angius@irgb.cnr.it

${ }^{5}$ Department of Biomedical Science, University of Sassari, Sassari, Italy

${ }^{6}$ Institute of Genetic and Biomedical Research, National Research Council

(CNR), Cittadella Universitaria di Cagliari, 09042 Monserrato, Cagliari, Italy

Full list of author information is available at the end of the article
}

(c) The Author(s). 2019 Open Access This article is distributed under the terms of the Creative Commons Attribution 4.0 International License (http://creativecommons.org/licenses/by/4.0/), which permits unrestricted use, distribution, and reproduction in any medium, provided you give appropriate credit to the original author(s) and the source, provide a link to the Creative Commons license, and indicate if changes were made. The Creative Commons Public Domain Dedication waiver (http://creativecommons.org/publicdomain/zero/1.0/) applies to the data made available in this article, unless otherwise stated. 


\section{Background}

Whole Exome Sequencing (WES) technology introduces a remarkable revolution in the identification of disease-causing genes and a powerful tool for genetic diagnosis, mainly relevant for rare diseases, which is remarkably difficult for clinicians to be diagnosed. WES can substantially reduce the number of cases remaining undiagnosed for many years and has become the current standard for the diagnosis of highly heterogeneous rare disorders with suspected Mendelian inheritance. This approach is leading to better dissecting the clinical phenotype of patients, in particular, those related to phenotypic progression in association with a single locus and those derived from mixed phenotypes developing from multilocus genomic variants. Recent studies reported the presence of multiple genomic diagnoses in a single individual in $3.2-7.2 \%$ of cases [1-3].

Molecular variants in the SCN9A gene (MIM\#603415) are responsible for a range of seizure disorders, which are characterized by early-onset isolated febrile seizures to generalized epilepsy with febrile seizures plus, type 7 (GEFS+), which identifies the most severe phenotype, as well as primary erythermalgia, callousness to pain linked to channelopathy and extreme paroxysmal pain disorder. Patients with isolated febrile seizures frequently showed an early onset between ages 5 months to 4 years and exhibit natural remission by age 6 years, while patients with GEFS+ persist in having various types of febrile and afebrile crises afterwards in life [4]. The SCN9A gene encodes for NaV1.7, a voltage-gated sodium channel mainly expressed in the hippocampus during the embryonic phase, suggesting a key function in the central nervous system [5] and in nociception signaling. SCN9A has been proposed suggested as a genetic modifier in SCN1A mutation linked with GEFS+ and as a potential susceptibility gene for Dravet syndrome $[6,7]$.

In 1975, KBG syndrome was identified and characterized by specific clinical findings: macrodontia of the upper central incisors, distinctive craniofacial signs, short stature, skeletal abnormalities and neurological involvement that encompasses developmental delay, convulsions and intellectual disability [8-11].

The initial description of the KBG syndrome, named KBG based on the initials of first affected families' surnames, referred to 7 patients from 3 unrelated families with a putative autosomal dominant inheritance $[8,12,13]$. To properly diagnose KBG syndrome, 4 or more of these 8 major criteria should be satisfied: (1) macrodontia of the upper central incisors, observed as a distinctive trait of KBG syndrome and reported in more than 95\% of cases; (2) distinctive facial features (presence of at least three findings of six categories of craniofacial shape, hair/eyebrow, eyes, ears, nose and mouth); 3) hand abnormalities, (fifth finger clinodactyly, clinical brachydactyly, or short tubular bones on radiographic exam); (4) neurological implication, with magnetic resonance, global developmental delay, and/or a seizure disorder; (5) bone age $>2 \mathrm{SD}$ below the average; (6) costovertebral abnormalities, (abnormal curvature of the spine, cervical ribs, or vertebral/endplate defects); (7) postnatal short stature (as a height less than 3rd centile); and (8) occurrence of a first-degree relative affected by KBG syndrome.

In 2011, Sirmaci et al., [14] identified pathogenic heterozygous variants in the ANKRD11 gene (MIM\# 611192). Single nucleotide mutations and small indels represent about $83 \%$ of the pathogenic variants identified within ANKRD11 and larger copy number variants (mostly deletions) represent about 17\% [15-17].

ANKRD11 represents one of the family members of ankyrin repeat-containing cofactors that relates with p160 nuclear receptor coactivators (NCOA1) by recruiting histone deacetylases to inhibit ligand-dependent transcriptional activation $[14,18,19]$. ANKRD11 was also found to localize inside the neurons nuclei and to accumulate in distinct inclusions after their depolarization. This finding suggests that ANKRD11 represents one of the major players in neural plasticity [14].

Here, we report two sisters from a non-consanguineous family, presenting generalized epilepsy with febrile seizures plus (GEFS+; MIM\#613863) associated with an heterozygous mutation in the SCN9A gene, p.(Lys655Arg), inherited from the father that was asymptomatic for the crisis, and a novel de novo loss of function mutation in the exon 10 of the ANKRD11 gene, p.(Tyr1715*) in one sister with a clinical phenotype compatible with KBG syndrome (MIM\#148050).

\section{Case presentation \\ Clinical data}

The reported family comes from Brazil and the two probands share a GEFS+ phenotypic spectrum. The original purpose of our study was to find the molecular causes of such phenotype. All members of the family were clinically assessed and diagnosed by the respective clinical neurologist and geneticist.

\section{Case II-1}

Female 14.5 years old. Delivered full term, by emergency $\mathrm{C}$-section, due to lack of fetal movement, weighing 2.971 $\mathrm{Kg}, 48 \mathrm{~cm}$ long and $33 \mathrm{~cm}$ head circumference. Neonatal period had no complications. From the neonatal period onward, she showed difficulty to breastfeed, with low weight gain. At 9 months old, she had myoclonus-atonic type seizures with sudden falling of the head and trunk. Initially precipitated by fever, these seizures became afebrile and daily, several times a day, and were controlled after substituting phenobarbital for sodium 
valproate (VPA), in low doses. The EEG tests initially showed focal spikes (centro-temporal regions) and only at 4 age, one EEG test showed a theta rhythm $(4-5 \mathrm{~Hz})$ in the temporo-occipital regions (T5-O1; T6-O2). At the age of 4 years and 8 months, after remission of seizures for 3 years, and normal EEG tests, VPA was suspended. Starting from 6 years of age, the EEG tests showed persistence of several bursts of irregular generalized polyspike-wave (PSW) and spike-wave discharge (SW), lasting 1-3 s. (Fig. 1a-p). Despite persisting abnormal EEGs, patient has not presented relapse of seizures and is not on medication.

Patient presented with recurrent otitis episodes and developed conductive hearing loss in left ear. A computed tomography scan of the mastoid showed signs of otomastoiditis in the left ear with obliteration of Prussak's space and cholesteatomatous process. Orthodontic evaluation conducted at 8 years of age showed dolichofacial pattern, maxillary protrusion, absence of lip seal, delayed eruption of permanent teeth, besides size increase of upper central incisors, with extra mamelar structures and whitish material of incisors and other teeth, compatible with hypoplasia (Fig. 2a, b). Cone-beam computed tomography of right oral lower-posterior region at 14.5 years of age, revealed dental units partially erupting and the presence of mixed-aspect images located between the dental roots, suggesting bone dysplasia (Fig. 2). The skeletal X-ray

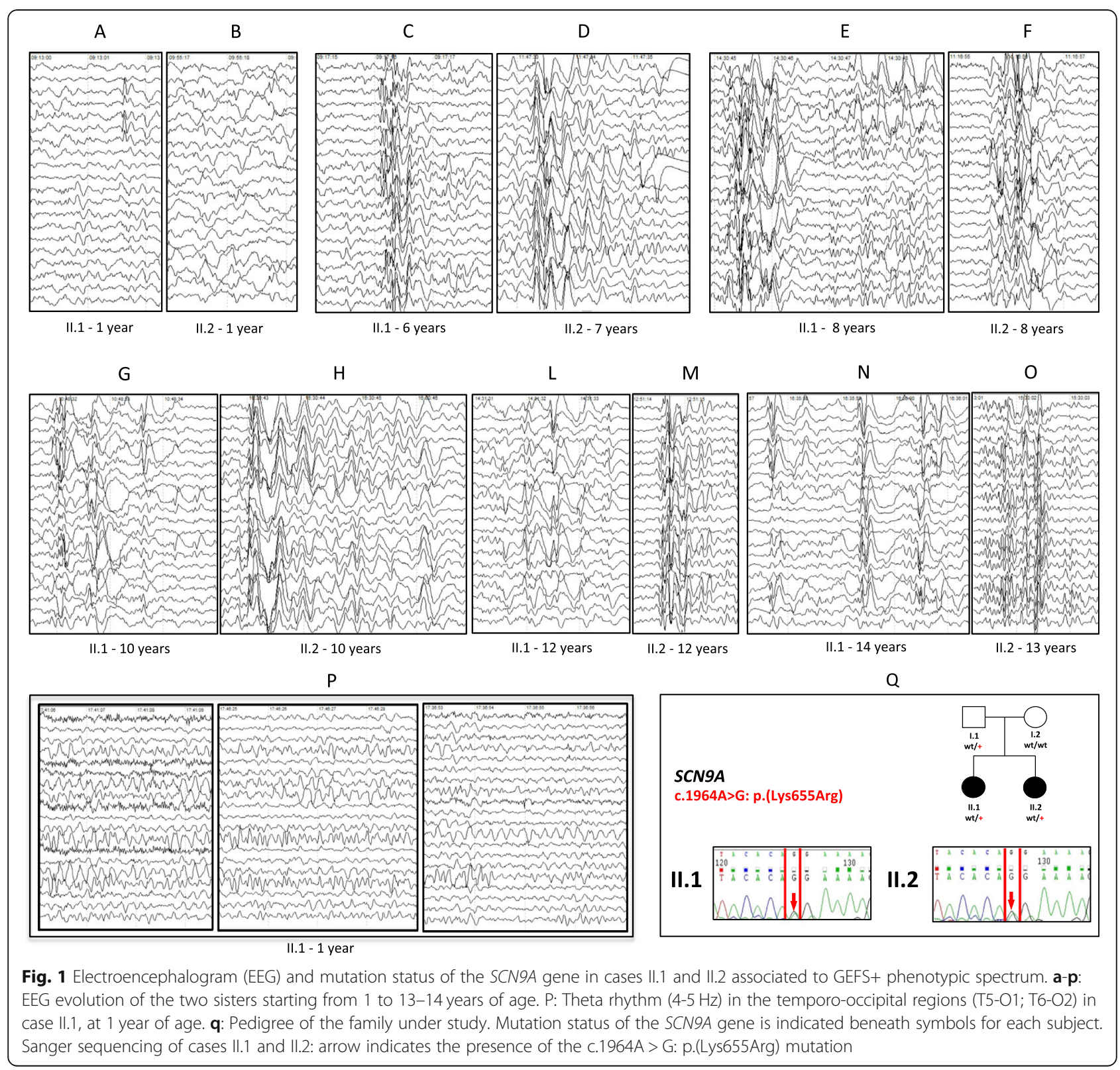



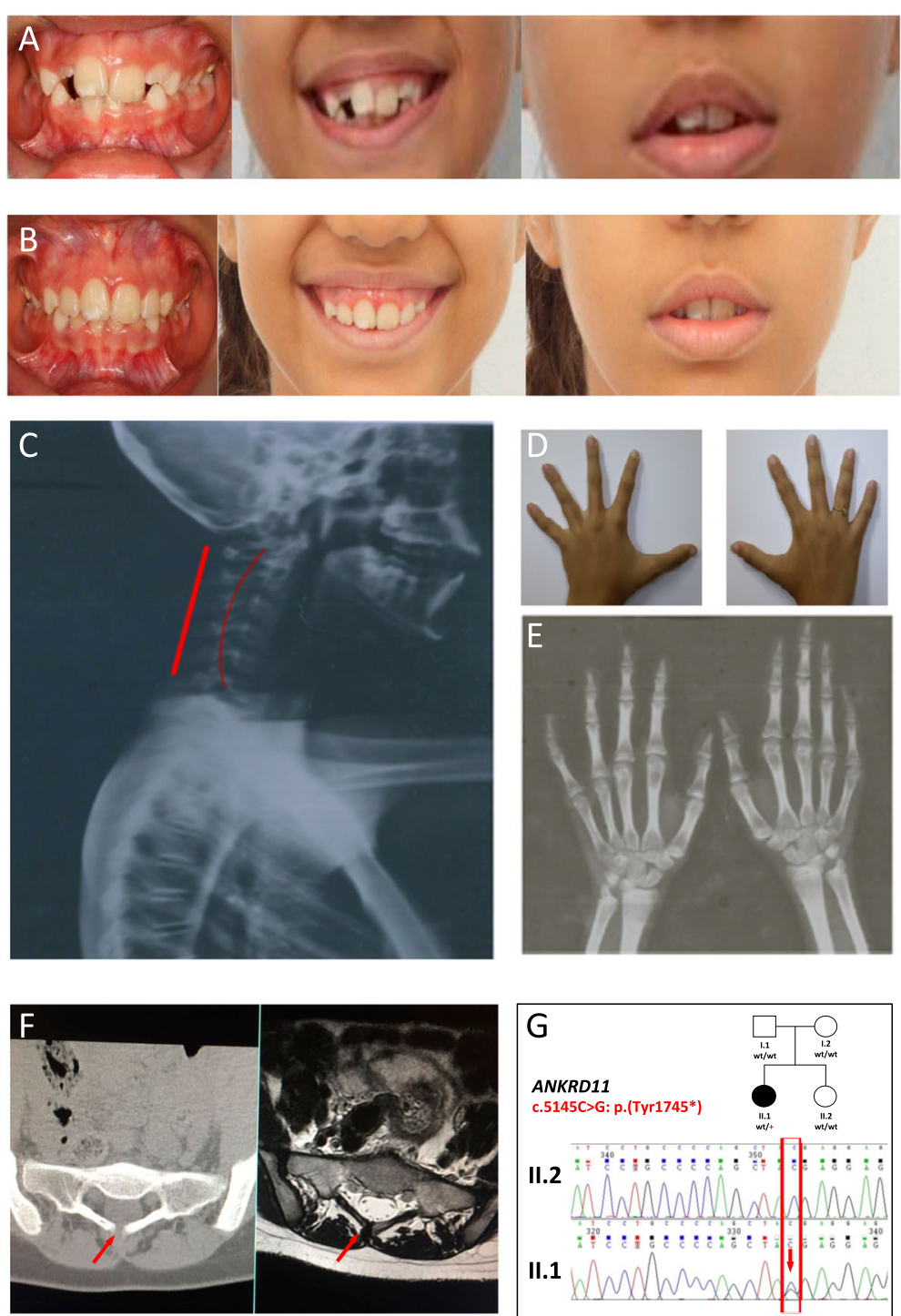

Fig. 2 Clinical features of case II.1 carrying the ANKRD11 mutation associated to the KBG syndrome. $\mathbf{a}$ and $\mathbf{b}$ : intra and extra oral views at 8 and 10 years, respectively. Due to the dental apparatus (an expander to enlarge the palate positioned at the age of about 8 and a half years), it was not possible to confirm the patient's sub-mucosal palate with magnetic resonance imaging. The clinical suspicion is based on the dentist's assessment of the palate. $\mathbf{c}$ : X-rays view of the cervical spine presenting invasion of physiological cervical lordosis. $\mathbf{d}$ and $\mathbf{e}$ : Picture and X-rays of patient hands demonstrating shortening of the distal phalanx of the 5th finger, clinodactyly of the 2th and 5th with a slight shortened tubular bones III and IV. f: Computed tomography and magnetic resonance imaging of the sacroiliac joints that allowed the display of concealed spina bifida at L5/S1. g: Pedigree of the family under study. Mutation status of the ANKRD11 gene is indicated beneath symbols for each subject. Sanger sequencing of cases II.1 and II.2: arrow indicates the presence of the C.5145C > G: p.(Tyr1745*) mutation

assessment showed inversion of physiological cervical lordosis (Fig. 2c); deviation of left dorsal axis, accentuated thoracic and lumbar lordosis and concealed spina bifida at L5/S1 (Fig. 2f). The proband has also shortening of the distal phalanx of the 5 th finger, clinodactyly of the 2th and 5th (Fig. 2d, e); myopia; bifid uvula with submucous cleft palate; weight and height growth curve below percentile $<5$. Neuropsychological analysis at age 8 showed IQ of 73 .

\section{Case II-2}

Female 13 years old. Showed normal neurological development. At 12 months, she had the first febrile seizure. She had recurring febrile seizures kept under control with low doses of VPA. After 3.8 years with no relapse of seizures and normal EEGs, VPA was suspended. At age 8 , the same electroencephalographic pattern observed in her sister appeared on the EEG (Fig. 1 a-p), with persistence of bursts of irregular generalized polyspike-wave 
(PSW) and spike-wave discharge (SW), less frequent and with shorter extent, with no relapse of seizures and no medication. She presents normal neuro-psychomotor and weight-height development, and an absence of dysmorphic and radiologic alterations.

\section{Genetic analysis}

Peripheral blood lymphocytes were collected from all affected individuals and their parents, and genomic DNA was extracted using a salting out procedure [20].

An array based Comparative Genomic Hybridization (CGH) analysis was done using commercially available Human Genome CGH Microarray (Agilent Technologies, Waldbronn, Germany) with an estimated average resolution of 13Kb (SurePrint G3 Human CGH Microarray).

NGS panel analysis was performed by Ampliseq/Ion Torrent technology with at least 120X using a targeted re-sequencing of 21 genes implicated in juvenile forms of epilepsy (ARHGEF9, KCNQ2, PRRT2, PNKP, ST3GAL3, SCN1A, GRIN2A, SCN8A, SLC2A1, SPTAN1, SCN2A, ALDHTA1, PCDH19, ARX, TBC1D24, KCNT1, PLCB1, STXBP1, PNPO, CDKL5, SLC25A22). No pathogenic variant has been identified with this method in our siblings.

One hundred nanograms of genomic DNA was used for DNA library preparation and exome enrichment using the Nextera Rapid Capture Expanded Exome Kit (Illumina) according to manufacturer instructions. DNA1000 chips (Agilent) and Qubit dsDNA BR Assay Kits (Invitrogen) were used to assess the quality of libraries. An indexed paired-end sequencing run $(101+7$ + 101 bp) was performed on a HiSeq 2000 using SBS Kit v3 chemistry (Illumina).

Using an analysis pipeline implemented in Orione [21], we performed read alignment to the human reference genome (hg19) using the Burrows-Wheeler Aligner 7 (BWA-MEM; version 0.7.5a) and GATK framework (version 2.8.1). Using GATK Unified Genotyper and GATK Variant Annotator modules, we annotated the variants as known or novel based on dbSNP146 and SnpSift/ SnpEff and KGGSeq. We used different models (SIFT, Polyphen2, LRT, MutationTaster, MutationAssessor and FATHMM) to assess the functional predictions for the aminoacid changes. We filtered the identified variants according to recessive/dominant/de novo pattern of inheritance, gene features and $\mathrm{MAF}<1 \%$ using as references dbSNP138, dbSNP141, 1000 Genomes, ESP6500, ExAC, gnomAD and EVADE, our private database of about 600 exomes). Subsequently, variants were evaluated for their phenotypic and biological impact.

The average target coverage was 93.5, 83.6, 88.1 and 78.9 for father, mother, case II-1 and case II-2, respectively. The target region was covered at least 10X in $93 \%$ for the father, $92 \%$ for the mother, $93 \%$ the older sister and $92 \%$ the younger. After filtering of the variants and the quality assessment in IGV browser, we identified a SCN9A: NM_002977:c.1964A > G: p.(Lys655Arg) (rs121908919); chr2:167138296 T/C in hg19 variant in both cases, inherited from an unaffected father. Additionally, we found a novel de novo truncating mutation in exon 10 of the ANKRD11 gene: NM_001256182:c.5145C > G: p.(Tyr1715*); chr16:89347805 G/C in hg19 in case II-1 (Figs. 1 and 2).

\section{Discussion and conclusions}

We report here a dual diagnosis in case II-1 presenting with ANKRD11 and SCN9A pathogenic variants found by WES. The variant in ANKRD11 has never been reported so far in literature. Considering that it is de novo, we supposed a possible mosaicism in the parents but we were not able to find any by WES analysis. The reassessment of phenotypic features in case II-1 confirmed that she fulfilled the proposed diagnostic criteria for KBG syndrome, complicated by early-onset isolated febrile seizures, although EEG abnormalities with or without seizures have been reported in some KBG cases [17]. In particular, she presented with macrodontia, hand anomalies, neurological involvement, costovertebral anomalies and post-natal short stature. This is the first Brazilian case reported so far.

The KBG syndrome is very rare, with about 100 individuals reported so far in literature $[15,16]$. Probably it is underdiagnosed due to the clinical features that can be mild and common to other diseases. ANKRD11 mutations have been found in patients identified in a large number of subjects with characteristics consistent with Cornelia de Lange syndrome (see, for example, CDLS1 MIM\#122470), thus showing phenotypic overlap between the two disorders. As previously reported [22, 23], some KBG patients could be recognized by gestalt, others may look like Cornelia de Lange syndrome (CDLS). CDLS and KBG represent two rare and distinct syndromes, but they have clinical aspects that overlap such as cognitive deficit, growth retardation and certain craniofacial abnormalities (brachycephaly, wide eyebrows and nostrils anti-vertite). Other common features are limb abnormalities such as small hands and feet, clinodactyly of the fifth finger and syndactyly of the second and third toes. Five different genes associated with the cohesin complex and its regulation (NIPBL, SMC1A, $S M C 3, H D A C 8$ and RAD21), showed heterozygous mutations identified in patients with CDLS. The cohesin complex regulate gene expression mediating transcriptional activation and repression [23]. The main function of ANKRD11 is to suppress transcriptional activation of the target genes of nuclear receptors by enrolling deacetylase at different promoters [18]. It is reasonable to assume that dysregulation of functionally correlated 
genes by cohesin complex deficiency or ANKRD11 may result in overlapping phenotypic characteristics [23].

The shared variant p.(Lys655Arg), occurring in $S C N 9 A$, has been previously found in several individuals: one with GEFS+ and two with Dravet syndrome. Additionally, one of these individual also possesses a de novo SCN1A mutation [6] and another affected by atypical benign partial epilepsy (ABPE) of childhood, harbored variants in in the CPA6 and SCNM1 genes associated with epilepsy [24]. Although already shown to play important pathogenic roles in epilepsy and predicted to significantly alter protein function, the p.(Lys655Arg) variant was detected in asymptomatic parents and has been found with extremely low frequency in control cohorts (NHLBI GO Exome Sequencing Project and ExAC Browser).The SCN9A p.Lys655Arg variant found in our sisters seems to be associated with an early-onset isolated febrile seizures. Other subjects showing febrile seizures or GEFS+, that possess SCN9A mutations were sporadic and cannot offer powerful evidence for a specific role of $S C N 9 A$ in seizure disorders that is presently under debate. Therefore, this report would further support that $S C N 9 A \mathrm{mu}$ tations are linked to a monogenic pattern.

The 39-year-old father, carrier of the same SCN9A variant, has not reported any history of seizures. Considering that most febrile seizures would naturally remit with age, we reinvestigated the family but we did not find evidence of history of seizure disorders in the father. However, he has had attention-deficit hyperactivity disorder since childhood and his family history shows neuropsychiatric disorders (dementia, schizophrenia, personality disorder, panic disorder, depression, delayed development, intellectual disability and autism spectrum disorder).

The phenotype of patients with GEFS+ combine febrile seizures, absence seizures, partial seizures, myoclonic seizures, or atonic seizures, with a variable degree of severity [25]. In a recent paper [7], none of the GEFS + families analyzed could be completely clarified by high penetrance of SCN9A mutations. Moreover, it is not surprising to identify a non-penetrant individual because they are commonly identified in autosomal dominant diseases and well documented in pedigrees of febrile crises (60-80\% of penetrance [6]).

This study provides an example of how WES has been instrumental allowing us to dissect the clinical phenotype, which is a multilocus variation aggregating in one proband. The successful identification of causal variant in a gene may not be sufficient, making it necessary to identify other variants that can fully explain the clinical picture. In several series of studies, the presence of multiple molecular diagnoses in a single individual has been described in $3.2-7.2 \%$ of cases that underwent molecular analysis, but large cohorts of patients and its associated clinical studies [3] are lacking to clearly define this phenomenon. This report emphasizes the critical role of the clinician in diagnostic genomic analyses and highlights the advantages of WES technology in the genetic dissection of a heterogeneous phenotype.

In our study, we prove that apparent phenotypic expansion may represent blended phenotypes resulting from pathogenic variation at more than one locus, so allowing a dissection of genotype-phenotype relationships. Due to the lack of the prevalence of blended phenotypes from multiple monogenic disorders, a systematic re-analysis of WES data sets is needed to a proper diagnosis in daily practice.

\section{Abbreviations}

ANKRD1: Ankyrin Repeat Domain 11; Array based CGH: Microarray based comparative genomic hybridization; CDLS: Cornelia de Lange syndrome; CPA6: Carboxypeptidase A6; EEG: electroencephalogram; GEFS: generalized epilepsy with febrile seizures; HDAC8: Histone Deacetylase 8; IQ: intelligence quotient; KBG: syndrome; L5/S1: Lumbar5/Sacral1 Junction; NaV1.7: Nav1.7, sodium channel encoded by SCN9A; NCOA1: nuclear receptor coactivator 1; NGS: Next Generation sequencing; NIPBL: Nipped-B-like protein gene; PSW: polyspike-wave; RAD21: RAD21 Cohesin Complex Component; SCN9A: Sodium Voltage-Gated Channel Alpha Subunit 9; SCNM1: Sodium channel modifier 1; SMC1A: structural maintenance of chromosomes 1A; SMC3: Structural maintenance of chromosomes protein 3; SW: spike-wave discharge; VPA: sodium valproate; WES: Whole exome sequencing

\begin{abstract}
Acknowledgments
We are sincerely grateful to the family for participating in this study. All of the authors would like to thank the CRS4 HPC group for IT support and computational infrastructure and Leonardo Barata of the Clinic Ponto Alto diagnostic by image for support in image analysis.
\end{abstract}

Funding

No funding was obtained for this study.

Availability of data and materials

The data of the current study are available from the corresponding author on reasonable request.

\section{Authors' contributions}

MFV, FCRZ, HPP, LCNV, MVAP and MBPT were responsible for acquisition of the clinical information and instrumental evaluation of patients. RA, AA and LC conceived and designed the experiments. MO, GP and IP performed the experiments. AA, PU, SO, GC and RA analyzed the data and performed statistical analysis. MBPT, RMA, FC, AA and LC jointly supervised research. RA, $A A$ and $L C$ wrote the paper with input from all coauthors. All authors read and approved the final version of the manuscript.

\section{Ethics approval and consent to participate}

The study protocol was performed in accordance with the Declaration of Helsinki and approved by the Ethics and Research Committee of the University Hospital Professor Edgard Santos in Bahia, Brasil (CEP/HUPES opinion No 121/2013)

\section{Consent for publication}

Written informed consent to publish medical information and images, reported in this publication, was obtained from all patients and/or parents of patients when not over 18 years old.

Competing interests

The authors declare that they have no competing interests. 


\section{Publisher's Note}

Springer Nature remains neutral with regard to jurisdictional claims in published maps and institutional affiliations.

\section{Author details}

'Postgraduate Program in Interactive Processes of Organs and Systems Federal University of Bahia, Salvador, Brazil. ${ }^{2}$ Research group Epi-Genétic, Salvador, Bahia, Brazil. ${ }^{3}$ Centre for Advanced Studies, Research and Development in Sardinia (CRS4), Science and Technology Park Polaris, Pula, Italy. ${ }^{4}$ EEG Service and Clinical Outpatient of Epilepsy, University Hospital Complex Professor Edgard Santos (C-HUPES), Federal University of Bahia, Salvador, Bahia, Brazil. ${ }^{5}$ Department of Biomedical Science, University of Sassari, Sassari, Italy. ${ }^{6}$ Institute of Genetic and Biomedical Research, National Research Council (CNR), Cittadella Universitaria di Cagliari, 09042 Monserrato, Cagliari, Italy. ${ }^{7}$ Clinic Ponto Alto diagnostic by Image, São Marcos, Salvador, Bahia, Brazil. ${ }^{8}$ Faculty of Medicine of the ABC, São Paulo, Brazil.

Received: 15 October 2018 Accepted: 3 January 2019

Published online: 14 January 2019

\section{References}

1. Posey JE, Rosenfeld JA, James RA, Bainbridge M, Niu Z, Wang X, et al. Molecular diagnostic experience of whole-exome sequencing in adult patients. Genet Med. 2016;18:678-85. https://doi.org/10.1038/gim.2015.142.

2. Balci TB, Hartley T, Xi Y, Dyment DA, Beaulieu CL, Bernier FP, et al. Debunking Occam's razor: diagnosing multiple genetic diseases in families by whole-exome sequencing. Clin Genet. 2017;92:281-9. https://doi.org/10. 1111/cge.12987.

3. Posey JE, Harel T, Liu P, Rosenfeld JA, James RA, Coban Akdemir ZH, et al. Resolution of disease phenotypes resulting from multilocus genomic variation. N Engl J Med. 2017;376:21-31. https://doi.org/10.1056/NEJMoa1516767.

4. Singh R, Scheffer IE, Crossland K, Berkovic SF. Generalized epilepsy with febrile seizures plus: a common childhood-onset genetic epilepsy syndrome. Ann Neurol. 1999;45:75-81.

5. Mechaly I, Scamps F, Chabbert C, Sans A, Valmier J. Molecular diversity of voltage-gated sodium channel alpha subunits expressed in neuronal and non-neuronal excitable cells. Neuroscience. 2005;130:389-96. https:/doi.org/ 10.1016/j.neuroscience.2004.09.034.

6. Singh NA, Pappas C, Dahle EJ, Claes LRF, Pruess TH, De Jonghe P, et al. A role of SCN9A in human epilepsies, as a cause of febrile seizures and as a potential modifier of Dravet syndrome. PLoS Genet. 2009;5:e1000649. https://doi.org/10.1371/journal.pgen.1000649.

7. Mulley JC, Hodgson B, McMahon JM, Iona X, Bellows S, Mullen SA, et al. Role of the sodium channel SCN9A in genetic epilepsy with febrile seizures plus and Dravet syndrome. Epilepsia. 2013;54:e122-6. https://doi.org/10. 1111/epi.12323.

8. Herrmann J, Pallister PD, Tiddy W, Opitz JM. The KBG syndrome-a syndrome of short stature, characteristic facies, mental retardation, macrodontia and skeletal anomalies. Birth Defects Orig Artic Ser. 1975;11:7-18.

9. Zollino M, Battaglia A, D'Avanzo MG, Della Bruna MM, Marini R, Scarano G, et al. Six additional cases of the KBG syndrome: clinical reports and outline of the diagnostic criteria. Am J Med Genet. 1994;52:302-7. https://doi.org/ 10.1002/ajmg.1320520310

10. Brancati F, D'Avanzo MG, Digilio MC, Sarkozy A, Biondi M, De Brasi D, et al. KBG syndrome in a cohort of Italian patients. Am J Med Genet A. 2004;131:144-9. https://doi.org/10.1002/ajmg.a.30292.

11. Skjei KL, Martin MM, Slavotinek AM. KBG syndrome: report of twins, neurological characteristics, and delineation of diagnostic criteria. Am J Med Genet A. 2007:143A:292-300. https://doi.org/10.1002/ajmg.a.31597.

12. Tekin M, Kavaz A, Berberoğlu M, Fitoz S, Ekim M, Ocal G, et al. The KBG syndrome: confirmation of autosomal dominant inheritance and further delineation of the phenotype. Am J Med Genet A. 2004;130A:284-7. https://doi.org/10.1002/ajmg.a.30291.

13. Fryns JP, Haspeslagh M. Mental retardation, short stature, minor skeletal anomalies, craniofacial dysmorphism and macrodontia in two sisters and their mother. Another variant example of the KBG syndrome? Clin Genet. 1984;26:69-72

14. Sirmaci A, Spiliopoulos M, Brancati F, Powell E, Duman D, Abrams A, et al. Mutations in ANKRD11 cause KBG syndrome, characterized by intellectual disability, skeletal malformations, and Macrodontia. Am J Hum Genet. 2011;89:289-94. https://doi.org/10.1016/j.ajhg.2011.06.007.
15. Goldenberg A, Riccardi F, Tessier A, Pfundt R, Busa T, Cacciagli P, et al. Clinical and molecular findings in 39 patients with KBG syndrome caused by deletion or mutation of ANKRD11. Am J Med Genet Part A. 2016;170: 2847-59. https://doi.org/10.1002/ajmg.a.37878.

16. Low K, Ashraf T, Canham N, Clayton-Smith J, Deshpande C, Donaldson A, et al. Clinical and genetic aspects of KBG syndrome. Am J Med Genet Part A. 2016;170:2835-46. https://doi.org/10.1002/ajmg.a.37842.

17. Morel Swols D, Foster J, Tekin M. KBG syndrome. Orphanet J Rare Dis. 2017;12:183. https://doi.org/10.1186/s13023-017-0736-8.

18. Zhang SS-M, Wei J, Qin H, Zhang L, Xie B, Hui P, et al. STAT3-mediated signaling in the determination of rod photoreceptor cell fate in mouse retina. Invest Ophthalmol Vis Sci. 2004;45:2407-12.

19. Zhang A, Li C-W, Chen JD. Characterization of transcriptional regulatory domains of ankyrin repeat cofactor-1. Biochem Biophys Res Commun. 2007:358:1034-40. https://doi.org/10.1016/j.bbrc.2007.05.017.

20. Miller SA, Dykes DD, Polesky HF. A simple salting out procedure for extracting DNA from human nucleated cells. Nucleic Acids Res. 1988;16: 1215.

21. Cuccuru G, Orsini M, Pinna A, Sbardellati A, Soranzo N, Travaglione A, et al. Orione, a web-based framework for NGS analysis in microbiology. Bioinformatics. 2014;30:1928-9. https://doi.org/10.1093/bioinformatics/ btu135.

22. Ansari M, Poke G, Ferry Q, Williamson K, Aldridge R, Meynert AM, et al. Genetic heterogeneity in Cornelia de Lange syndrome (CdLS) and CdLS-like phenotypes with observed and predicted levels of mosaicism. J Med Genet. 2014:51:659-68. https://doi.org/10.1136/jmedgenet-2014-102573.

23. Parenti I, Gervasini C, Pozojevic J, Graul-Neumann L, Azzollini J, Braunholz D, et al. Broadening of cohesinopathies: exome sequencing identifies mutations in ANKRD11 in two patients with Cornelia de Lange-overlapping phenotype. Clin Genet. 2016;89:74-81. https://doi.org/10.1111/cge.12564.

24. Allen NM, Conroy J, Deonna T, McCreary D, McGettigan P, Madigan C, et al. Atypical benign partial epilepsy of childhood with acquired neurocognitive, lexical semantic, and autistic spectrum disorder. Epilepsy Behav Case Reports. 2016;6:42-8. https://doi.org/10.1016/j.ebcr.2016.04.003.

25. Wallace $\mathrm{RH}$, Wang DW, Singh $\mathrm{R}$, Scheffer IE, George AL, Phillips HA, et al. Febrile seizures and generalized epilepsy associated with a mutation in the Na+-channel B1 subunit gene SCN1B. Nat Genet. 1998;19:366-70. https:// doi.org/10.1038/1252.
Ready to submit your research? Choose BMC and benefit from:

- fast, convenient online submission

- thorough peer review by experienced researchers in your field

- rapid publication on acceptance

- support for research data, including large and complex data types

- gold Open Access which fosters wider collaboration and increased citations

- maximum visibility for your research: over $100 \mathrm{M}$ website views per year

At $\mathrm{BMC}$, research is always in progress.

Learn more biomedcentral.com/submissions 